\title{
TRANSFORMATION EINER EPK IN EINE FORMALISIERTE METHODE
}

\author{
Klaus D. Bösing, Jan Geldmacher
}

\section{Zusammenfassung}

Dieser Beitrag untersucht die Transformation einer Ereignisgesteuerten Prozesskette in diverse formalisierte Modellierungsmethoden. Die hierfür gewählten formalen Methoden sind Business Process Model and Notation, Unified Modeling Language, Subjektorientiertes Business Process Management und Petrinetze. Das Ziel dieser Arbeit ist es, die Vor- und Nachteile der Methoden zu definieren und eine geeignete Methode für sowohl Modellierung als auch Automatisierung von Geschäftsprozessen zu finden. Zunächst werden theoretische Grundlagen der Geschäftsprozessmodellierung dargestellt. Des Weiteren wird auf die gesonderten Charakteristika der vorgestellten Methoden eingegangen. Ein besonderer Fokus liegt hier auf dem Thema Syntax und Semantik der Methoden. Nachfolgend werden die Ergebnisse der Transformation sowie die Vorteile und Nachteile der gewählten Methoden kritisch betrachtet. Als Grundlage für den Vergleich wurde das Beispiel eines Bewerbungsverfahrens als Ereignisgesteuerte Prozesskette entwickelt.

\section{Abstract}

This paper examines the transformation of an event-driven process chain in the most used formal modeling methods for development of business processes. The chosen formal methods are Business Process Model and Notation, Unified Modeling Language, Subject-oriented Business Process Management and Petri nets. The aim of this paper is to define the advantages and disadvantages of these methods and to determine the most suitable method for modeling and automation of business processes that enables the transformation of an EPC into a formal method. The first phase of this paper consists of some basic theoretical topics about business process modeling. In the second phase an analysis of the special characteristics of the methods, such as syntax, semantics etc. can be found. The third part contains the results of the transformations, which is a comparison of EPC with each formal method and a determination of their advantages and disadvantages. For the comparison the EPC of the "application for employment" procedure was first developed. After that, the EPC was transformed into the four formal methods.

\section{EINLEITUNG}

Die Motivation für diesen Beitrag entstand in Projekten in Zusammenarbeit mit dem ICT-Dienstleistungsunternehmen T-Systems. In einer Zeit, in der sich Märkte schnell verändern und Unternehmen dem globalen Wettbewerb ausgesetzt sind, werden immer neue Wege gesucht, noch effektiver und effizienter zu wirtschaften, um am Markt bestehen zu können. Aber nicht nur der Wettbewerb wird härter, auch die Erwartungen der Kunden steigen. Schnelligkeit und Flexibilität sowie Kundennähe sind dabei oft genannte Forderungen. Unternehmen stehen dadurch im ständigen Wandel. Um auf diese Veränderungen am Markt reagieren zu können, ist es für sie unabdingbar, die betrieblichen Abläufe stetig zu optimieren bzw. an die Gegebenheiten anzupassen. Dafür ist jedoch eine Dokumentation sinnvoll, die es ermöglicht, die Prozessabläufe zu strukturieren und Standards festzuhalten. Prozesse, die im alltäglichen
Arbeitsgeschäft schon fast automatisch ablaufen, werden häufig nur von den Mitarbeitern beherrscht, die sie ausführen, und nicht selten wenden diese Mitarbeiter Standards an, die sie selbst durch ihre Erfahrung und Arbeit mit den Prozessen gesammelt und festgelegt haben. In der Praxis steht daher auch der Prozess und nicht die Technologie, die diesen ermöglicht, im Mittelpunkt. Die Geschäftsprozesse sind also maßgeblich am Erfolg eines Unternehmens beteiligt. Der Detaillierungsgrad eines Prozesses spielt eine ebenfalls wichtige Rolle, da eine neue Abstraktionsebene eines in der Realität bestehenden Prozesses dazu neigen kann, relevante Informationen auszulassen. Ohne eine Dokumentation der betrieblichen Abläufe ist es nicht möglich, komplexe Sachverhalte auf eine einheitliche Sicht zu reduzieren.

Mittlerweile gibt es eine Vielzahl von Dokumentationsmöglichkeiten, um betriebliche Abläufe transparent zu machen und eine Optimierung die- ser zu ermöglichen. Die Modellierung der Geschäftsprozesse kann das Verständnis über Tätigkeiten, Funktionen, Rollen oder Schnittstellen geben und somit zur Erhöhung der Transparenz von Abläufen innerhalb und außerhalb eines Unternehmens beitragen. Damit wird zum einen die Verfahrenssicherheit erhöht, die wiederum Risiken minimieren kann, und zum anderen schafft die Modellierung eine Basis für weiterführende Aktionen, wie z. B. Schwachstellenanalysen oder Optimierung organisatorischer Abläufe. Die Automatisierung ermöglicht nicht nur die Verwaltung der Arbeitsabläufe (Workflow-Management), sondern auch die Verkürzung von Prozessen. Eine ganzheitliche Optimierung betrieblicher Abläufe ist demnach in der Praxis nur durch die Modellierung und die Automatisierung dieser in Form von Geschäftsprozessmodellen möglich.

Die Modellierungsmethode Ereignisgesteuerte Prozesskette (EPK) hat sich nicht als formeller Standard durch- 
setzen können, wird aber dennoch in vielen Unternehmen als de-factoStandard betrachtet. Grundsätzlich erfüllt die Methode die fachlichen Anforderungen, die an die Prozessmodellierung gestellt werden. In der Praxis führt die Methode u. a. zu folgenden Problemen (Bösing 2013) :

- Bei der Abbildung von komplexen und kreativen Geschäftsprozessen lassen sich Überwachungs- und Kontrollstrukturen nur bedingt modellieren.

- Wegen der strengen Modellierungsregel gestaltet sich eine aussagekräftige Benennung von Ereignissen oft als problematisch.

- Die EPK-Methode ist für die Simulation von Geschäftsprozessen nur geeignet, wenn zusätzliche Informationen zur Verfügung gestellt werden.

Dies sind nur einige Beispiele. Heute besteht in vielen Unternehmen der Wunsch, ihre vielmals auf fachlicher Ebene modellierten Geschäftsprozesse als Workflow mit einem Workflow Management System ausführen zu lassen. Die Methode stößt aufgrund des fachlichen Konzepts an ihre Grenzen, da die Möglichkeit der Umsetzung in automatisierte Workflows fehlt. Aufgrund des fehlenden Formalismus stellt sich die Frage, inwieweit sich eine semiformale Methode in eine formalisierte Methode überführen lässt.

Ziel dieses Beitrags ist es, eine geeignete Methode zur Modellierung und Automatisierung von Geschäftsprozessen zu ermitteln, die die Transformation einer Ereignisgesteuerten Prozesskette (EPK) in eine formalisierte Methode ermöglicht. Diese soll sowohl fachlich als auch operativ einsetzbar sein und bestenfalls ohne Verluste von Informationen funktionieren. Zudem sollen die gebräuchlichen Methoden aus der Praxis vergleichend dargestellt werden, um Vor- und Nachteile aufzuzeigen und diese auf ihre Nachhaltigkeit in der Praxis zu prüfen. Darüber hinaus soll untersucht werden, welche formalen Methoden für den langjährigen Einsatz in Unternehmen tragbar und geeignet sind.

Grundlage dieses Beitrags ist das Ergebnis eines Projekts "Modellierung und Automatisierung von Geschäftsprozessen“ im Sommersemester 2013 im Masterstudiengang Wirtschaftsinformatik der TH Wildau, an dem Luisa Alexandrow, Jan Geldmacher als Projektleiter, Sofia Geraniotaki, Nikolai Paul und Ansgar Wilhelm beteiligt waren (Alexandrow et al. 2013).

\section{GRUNDLAGEN DER GESCHÄFTS- PROZESSMODELLIERUNG}

\section{II.I INFORMELLE, SEMIFORMALE UND FORMALE SPEZIFIKATION}

Jede Modellierungsmethode gehört zu einer Formalitätskategorie. Es gibt drei Formalitätskategorien: formale, semiformale und informelle Methoden (Abb. 1). Die Unterscheidung zwischen den drei Kategorien wird entsprechend der Definition der Syntax und der Semantik jeder Methode getroffen (Brocke 2003):

- Informelle Methoden haben weder eine definierte Semantik noch eine definierte Syntax.

- Semiformale Methoden haben eine definierte Syntax, aber ihre Semantik ist nicht eindeutig definiert.

- Formale Methoden haben eine definierte Semantik und Syntax.

\begin{tabular}{|c|c|c|}
\hline Methode & Syntax & Semantik \\
\hline Informelle Methode & nein & nein \\
\hline Semiformale Methode & ja & nicht eindeutig definiert \\
\hline Formale Methode & ja & ja \\
\hline
\end{tabular}

Abb. 1) Formalitäten von Modellierungsmethoden.

\section{II.II SYNTAX UND SEMANTIK}

Die Weitergabe von Informationen über eine Methode erfordert zwei grundlegende Dinge. Zum einen muss ein dem Sender und Empfänger bekannter Zeichensatz zur Verfügung stehen (Semantik) und zum anderen müssen beide Seiten die gleichen Regeln zum Einsatz dieser Zeichen verwenden (Syntax), damit die Information in einer Nachricht fehlerfrei übertragen werden kann.
Auch für die Modellierung und Automatisierung von Geschäftsprozessen müssen genau diese beiden Voraussetzungen erfüllt sein, weil ein Geschäftsprozess nichts anderes ist als eine Reihe von Informationen, die von einem Menschen zum anderen oder einer Software zur Automatisierung von Geschäftsprozessen übertragen werden.

Die Anforderungen an die Syntax und Semantik sind dabei umfassend. Beide sollen alle relevanten Informationen der Geschäftsprozesse übersichtlich, kurz und automatisierbar darstellen können. Die Auswahl der möglichen Symbole ist ein entscheidender Schritt bei der Komposition einer Methode, denn sie müssen aussagekräftig genug sein, um alle relevanten Informationen zu enthalten, dürfen jedoch nicht zu zahlreich werden, damit der Überblick nicht verloren geht. Der anspruchsvollste Teil ist jedoch die Möglichkeit der Automatisierung von Geschäftsprozessen. Denn in vielen Unternehmen besteht heute der Wunsch, Geschäftsprozesse als Workflows automatisch ausführen zu lassen (Bösing 2013). Damit ein Geschäftsprozess mittels einer Methode komplett automatisierbar ist, muss diese eine formale Semantik aufweisen (Eckleder 2006). Die meisten heutzutage angewandten Methoden werden in einer XML-Datei definiert und sind nicht formal. 
das Workflow-Management (operative Ebene).

Auf der strategischen Ebene erfolgt die Betrachtung der einzelnen Geschäftsfelder des Unternehmens und somit die Auseinandersetzung mit dem damit verbundenen Erfolgsfaktoren. Folge davon ist die Entwicklung von Strategien für das Prozessmanagement. Aus den entwickelten Strategien werden auf der fachlich-konzeptionellen Ebene Geschäftsprozesse abgeleitet. In dieser Ebene werden mögliche Varianten eines Prozesses bewertet und anschließend die geeignetste ausgewählt. Anschließend folgt die Modellierung der Prozesse. Auf der operativen Ebene werden im ersten Schritt die in der fachlich-konzeptionellen Ebene modellierten Prozesse um weitere technische Elemente erweitert. Dieser Schritt ist nötig, um den Prozess mittels eines Workflow-ManagementSystems automatisiert ausführen zu können. Die Ausführung dieser Prozesse ist der nächste Schritt in der operativen Ebene. Es werden konkrete Objekte des Prozesses erzeugt und in einem Workflow-Management-System ausgeführt. Im letzten Schritt, dem Prozess-Monitoring, wird das Verhalten der Prozesse verfolgt und kontrolliert (Gehring und Gadatsch 1999).

\section{II.IV MODELLIERUNGSMETHODEN}

Inzwischen gibt es eine Vielzahl von Methoden für die Modellierung von Geschäftsprozessen, die für unterschiedliche Aufgaben entwickelt wurden. Die Methoden lassen sich in verschiedene Formalitätskategorien einordnen und haben eine mehr oder weniger ausgeprägte Syntax und Semantik. Ferner können sie unterschiedlichen Ebenen der Unternehmensstrategie zugeordnet werden. In der Vergangenheit diente die Modellierung überwiegend der Dokumentation und Analyse von Geschäftsprozessen. Vornehmlich wurden semiformale Methoden wie beispielsweise Ereignisgesteuerte Prozessketten (EPKs) eingesetzt. In Unternehmen basieren viele in der Vergangenheit modellierte Geschäftsprozesse auf der EPK-Methode. Galt es in der Vergangenheit bestehende Prozesse systematisch zu dokumentieren, um sie anschließend analysieren zu können, steht heute die Automatisierung von Geschäftsprozessen deutlich im Vordergrund. Die Überführung von EPKs als semiformale Methode in eine formale Modellierungsmethode wie beispielsweise der Business Model und Notation (BPMN) könnte dem Wunsch vieler Unternehmen ein Stück näher kommen, ihre mit Hilfe der EPKs entwickelten Geschäftsprozesse als Workflows auszuführen zu lassen. Damit könnten die in der Vergangenheit investierten Kosten gerechtfertigt werden. Für die Transformation von EPKs in formale Methoden wurden inzwischen unterschiedliche Ansätze entwickelt. In (Decker et al. 2009) und (Kotsev et al. 2011) werden Ansätze der Transformation von EPKs in BPMNs unter der Bedingung beschrieben, dass beide Modelle vollständig konform sind und kein Informationsverlust entsteht. In dem Ansatz von (Decker et al. 2009) wird beispielsweise deutlich, dass nur Teilbereiche automatisch bzw. halb automatisch in BPMN-Modelle überführt werden können. In (Dandl 1999) wird dokumentiert, dass objektorientierte Modelle nicht alle Aspekte der prozessorientierten Modelle abdecken. Da die S-BPM-Methode relativ neu ist, gibt es derzeit keine konkreten Lösungsansätze zur Transformation der EPK- in die S-BPM-Methode.

\section{ANALYSE UND EINSATZ DER METHODEN IN DER PRAXIS}

\section{III.I MODELLIERUNG AM BEISPIEL EINES BEWERBUNGSVERFAHRENS}

Um Aussagen über eine mögliche Transformation der EPK-Methode in eine formalisierte Methode bestmöglich darstellen zu können, wird ein Modell benötigt, das nach Möglichkeit viele syntaktische Elemente enthält und über die jeweilig verwendete Semantik der einzelnen Methoden überführt werden kann. Im vorliegenden Fall erfolgt dies am Beispiel eines Bewerbungsverfahrens, sowohl aus der Sicht des Bewerbers/der Bewerberin als auch der des Unternehmens (Abb. 2):

Zunächst schreibt der/die Bewerber/in eine Bewerbung an das Unternehmen, welches diese über den Postweg erhält und daraufhin eine Eingangsbestäti- gung aufgibt und zurück an den Absender schickt. Anschließend prüft das Unternehmen die Bewerbung für diese Position und darüber hinaus auch für alle Stellen, auf die die Bewerbung eventuell zusätzlich passen könnte. Sollte die Bewerbung auf eine freie Stelle passen, so wird der/die Bewerber/in zu einem Interview eingeladen. Diese Einladung erfolgt wieder über den Postweg. Der/die Bewerber/in kann nach Erhalt der Einladung diese bestätigen oder einen anderen Termin vorschlagen. Die Antwort geht zurück zum Unternehmen, wo nun entweder der Termin genehmigt wird oder eine erneute Einladung mit einem anderen Termin bzw. eine Absage erteilt wird. Sollte eine Absage erteilt werden, so wird diese dem/der Bewerber/in mitgeteilt und der Prozess ist beendet. Sollte ein Interviewtermin beidseitig zustande kommen, so kann das Interview durchgeführt werden. Das Unternehmen kann nun entscheiden, ob es den/die Bewerber/in einstellen möchte oder nicht.

In diesem Beispiel werden alle Konnektoren (AND, OR, XOR) verwendet. Im Kontext ergeben sich bestimmte Ereignisse, die erst nach Ausführung bestimmter Ereignisse in Kraft treten. Es ist eine Interaktion von zwei Akteuren (Bewerber/in und Unternehmen) und bietet somit eine gute Ausgangslage zur Modellierung und Transformation in die jeweiligen Modelle.

\section{III.I.I EREIGNISGESTEUERTE PROZESSKETTEN}

Die Ereignisgesteuerte Prozesskette (EPK) des Bewerbungsverfahrens wurde mit Hilfe des ARIS-Express-Modellierungswerkzeugs entwickelt. Während der Entwicklung wurden einige Vorteile und Nachteile dieser Methode festgestellt.

Es ist schwierig, den Prozess an einigen Stellen nachzubilden, auch wenn die Syntax und die Semantik der EPKs einfach zu verstehen sind. Im Wesentlichen passiert das, weil viele Regeln gerade in puncto der bereits angesprochenen Konnektoren sich nicht eins zu eins abbilden lassen, was sehr zeitintensiv ist. Ein weiterer Nachteil ist, dass jeder Funktion eine Rolle zu- 


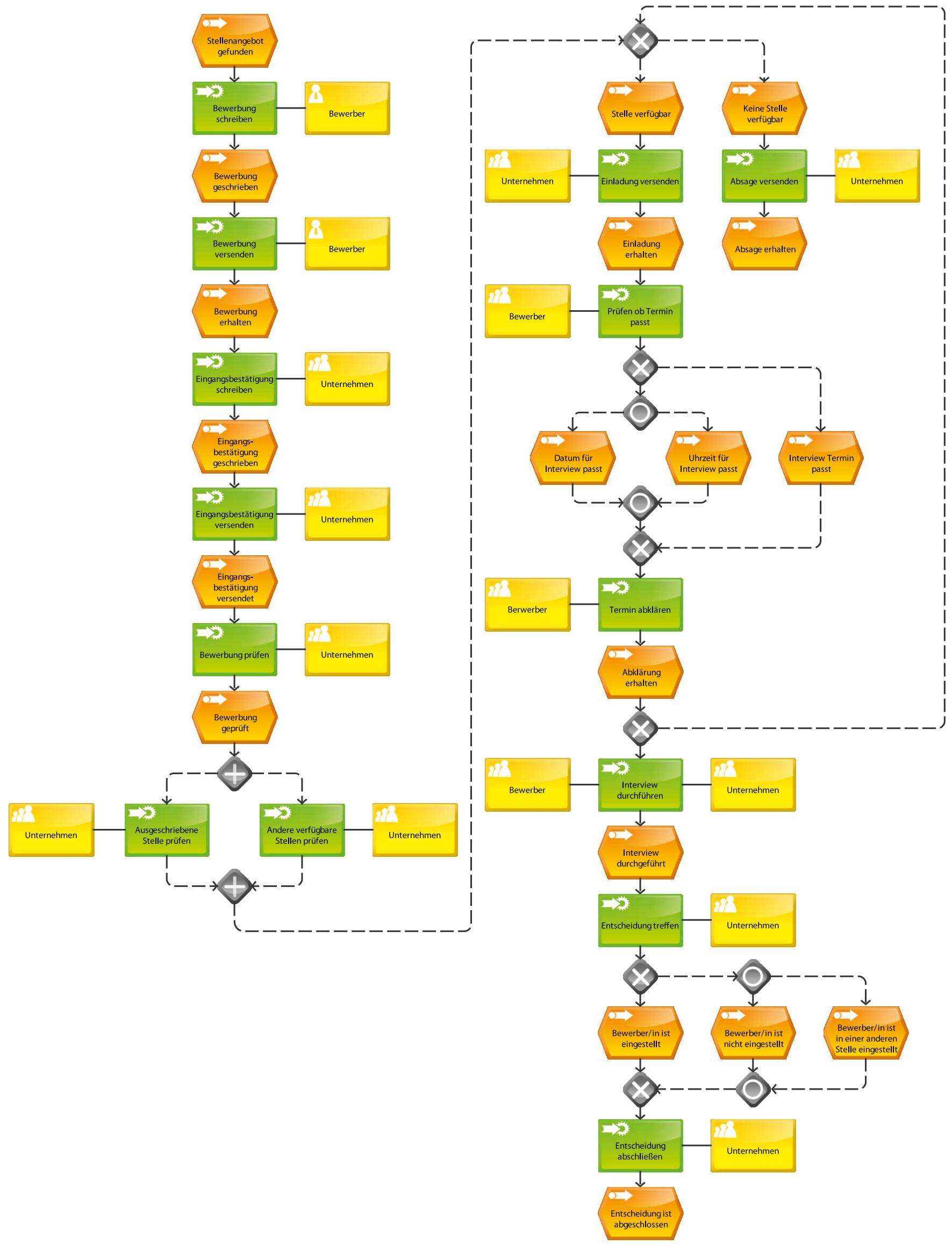

Abb. 2) Bewerbungsprozess als EPK-Basismodell modelliert. 
geordnet werden muss. Deswegen gibt es dieselbe Rolle mehrmals statt nur einmal, wie z. B. in BPMN. Teilweise ist es nötig, die EPK an einer Stelle zu ändern. Diese Stelle konnte nicht so einfach ausfindig gemacht werden, da EPKs sehr lang und komplex werden können. Abhilfe schaffen hier die sogenannten Vorgangskettendiagramme, in denen eindeutig zugewiesen werden kann, wo die Zuständigkeitsbereiche der involvierten Akteure liegen.

Die EPK-Methode ist mächtig, weil sie exakt so viele Elemente besitzt, wie zur Darstellung eines Prozesses benötigt werden. Trotzdem wäre es hilfreicher, wenn noch mehr syntaktische Elemente bereitgestellt werden würden. Dies könnte vor allem bei der Darstellung zu mehr Klarheit beitragen. Im Fall des Bewerbungsverfahrens wäre z. B. ein spezielles Symbol für zeitgesteuerte Aktivitäten hilfreich.

Die Abbildung des Bewerbungsverfahrens ist letztendlich unproblematisch, da das gewählte Szenario nur einen kleinen überschaubaren Prozess darstellt. Bei größeren Prozessen, wie sie in den meisten Unternehmen vorkommen, ist dies wiederum komplexer und zeitintensiver. Ein großer und wichtiger Nachteil der EPK-Methode ist, dass die modellierten Prozesse nicht automatisierbar sind.

\section{III.I.II SUBJEKTORIENTIERTES BPM}

Nach der Modellierung bzw. Transformation des Beispielszenarios in Subjektorientiertes BPM (S-BPM) kann festgestellt werden, dass S-BPM aufgrund der einfachen Verwendung mit lediglich fünf Elementen für die Prozessmodellierung - für jeden Anwender geeignet ist. Dieser kann ohne besondere IT-Kenntnisse oder auch im Bereich der Modellierung im Allgemeinen komplexe Geschäftsprozesse beschreiben und diese als Applikation direkt ausführen sowie ad hoc wieder ändern. Dies wird zudem durch die Orientierung am Aufbau der natürlichen Sprache ermöglicht. Allein diese Aspekte können für Unternehmen wirtschaftsfördernd sein, da beispielsweise Schulungen für Mitarbeiter nicht dringend notwendig werden. Für den Einstieg wird dem Mitarbeiter ein umfangreiches Handbuch zur Verfügung gestellt, das in ausführlichen und detaillierten Schritten die Methode und den Umgang mit der Software Metasonic Suite von der Metasonic AG beschreibt (Metasonic 2010).

Ein weiterer Punkt ist, dass dieses Modell durch die direkte Ausführung in Echtzeit dynamische Prozesse erlebbar machen kann. So ermöglicht S-BPM in Verbindung mit der Metasonic Suite eine sofortige Transformation in eine formale Theorie der Informatik. Die Prozessmodellierung ist dann für Maschinen lesbar bzw. kann diese als IT-Anwendung ausgeführt werden. Dies ist ein entscheidender Vorteil gegenüber der EPK-Methode, denn so muss das Prozessmodell nicht mehr in Software neu umgesetzt oder programmiert werden. S-BPM ist außerdem über eine erweiterte Applikation automatisierbar und somit in diesem Punkt bisher einzigartig.

Im Vergleich zu EPK stellt die S-BPMMethode die Kommunikation bzw. Interaktion mit den Beteiligten (Subjekten) in den Mittelpunkt und adressiert damit einen sehr wichtigen Aspekt der organisatorischen Unternehmenskultur. Die Akteure modellieren ihre Abläufe selbst anhand der intuitiv bedienbaren Benutzeroberfläche und steuern dazu die benötigen Services. Die Prozessbeschreibung wird als XML-Dokument abgelegt und bei Ausführung als Workflow interpretiert. Das Modell stellt somit die Grundlage der Prozessausführung dar. Somit lässt sich vor allem die eingangs in dieser Arbeit beschriebene Problematik der nicht gegebenen Transparenz von Prozessen deutlich mindern, da Mitarbeiter durch die Subjektsicht und einfache Bedienung der Methode von Anfang an in die Prozessgestaltung eingebunden werden. So kann dieser Aspekt zu mehr Motivation und Zufriedenheit in Unternehmen führen, da Mitarbeiter sich stärker mit dem Unternehmen und den Prozessen identifizieren und bemüht sind, sich kontinuierlich zu beteiligen sowie Verbesserungsvorschläge einzubringen.

Vergleichend zur EPK-Methode ist jedoch ein entscheidender Nachteil festzustellen. Bei der Prozesserstellung mittels S-BPM gibt es keine Konnektoren, wie es in EPK der Fall ist. Dies führt beim gewählten Szenario bereits zu einer unübersichtlichen Darstellung des Modells.

Im Fallbeispiel stellt sich heraus, dass nach dem Schritt der Bewerbungsprüfung entweder der Bewerber für die ausgeschriebene Stelle oder für eine andere verfügbare Stelle geprüft wird und daraufhin entweder eine Zusage oder eine Absage erhält. Zwar sind die gerichteten Graphen zu einem Funktionszustand in diesem Beispiel noch nachvollziehbar, die fehlenden Konnektoren führen aber bereits zur Unübersichtlichkeit des gesamten Modells. Daraus resultiert, dass bei größeren Prozessen, die in der Prozessmodellierung nicht selten anzutreffen sind, dieser Nachteil zu einem gravierenden Problem führen kann. Die Modellierung wird komplex und dies kann sogar bis zur Überforderung der Mitarbeiter führen, da diese die Prozesse selbst realisieren sollen, aber aufgrund der entstehenden Unübersichtlichkeit das Modell nicht mehr richtig lesen respektive verstehen können.

\section{III.I.III OBJEKTORIENTIERTE GESCHÄFTS- PROZESSMODELLIERUNG}

Aktivitätsdiagramme der Unified Modeling Language (UML) werden verwendet, um dynamische Aspekte eines Systems zu modellieren. Modelliert werden nacheinander ablaufende oder möglicherweise nebenläufig ablaufende Schritte im Verarbeitungsprozess. Die Konzentration bei der Modellierung dieser dynamischen Aspekte bezieht sich auf die Aktivitäten, die zwischen den Objekten stattfinden.

Eine Aktivität ist ein Zustand mit einer internen Aktion und einer oder mehreren ausgehenden Transitionen. Sie ist ein einzelner Schritt im Prozessablauf. Wenn Transitionen durch die Bedingungen unterschieden werden, können einer Aktivität auch mehrere Transitionen folgen. Besteht ein Prozess also aus vielen parallelen Aktivitäten, so wird das Modell aufgrund zahlreicher Synchronisationsknoten schnell unübersichtlich. Durch den Einsatz von Partitionen bzw. Pools kann das Modell zwar in sogenannte Aktivi- 
tätsbereiche unterteilt werden, die Darstellung bei komplexen Prozessen bleibt jedoch aufwendig.

\section{III.I.IV BUSINESS PROCESS MODEL AND NOTATION}

Grundsätzlich ist die Transformation eines EPK mittels der Business Process Model and Notation (BPMN) einfach, da schon mit den Grundbausteinen eine einfache Abbildung eines Geschäftsprozesses möglich ist. Diese Abbildung lässt sich nach Bedarf auch detaillierter darstellen. Durch die Standardisierung der Object Management Group (OMG) ist BPMN überall anwendbar und wird kontinuierlich weiterentwickelt. BPMN bietet eine optimale Verbindung zwischen Geschäftsprozessen und IT, die lediglich aufgrund der hohen Komplexität schwer zu erlernen ist. Sogenannte Pools und Swimlanes (Object Management Group 2010) zeigen auf einen Blick alle Zugehörigkeiten im Prozess und können über Markierungen und Annotationen zusätzliche Informationen erhalten. Generell wirkt ein Modell in BPMN deutlich übersichtlicher und strukturierter als ein vergleichbares EPK. Bei der automatisierten Transformation des Bewerbungsverfahrens (als EPK) traten zunehmend Fehler bei den (X)OR-Konnektoren auf, welche einer manuellen Nachbearbeitung bedürfen.

In einem Unternehmen ist eine direkte Einarbeitung der Belegschaft in die Symbolik von BPMN wünschenswert, da so der zusätzliche Schritt über ein EPK umgangen werden kann. Diese direkte Einarbeitung birgt jedoch aufgrund der Zeitintensität auch ein mögliches Risiko, da BPMN insgesamt aus über 150 verschiedenen Symbolen besteht (Object Management Group 2010). Bestimmte Geschäftsprozesse können unter Umständen anders abgebildet werden, da viele fachliche Aspekte fallbezogen sind und damit nicht berücksichtigt werden.

\section{III.I.IV PETRINETZE}

Petrinetze gelten allgemein als der Ursprung für Simulationstechnik und Verfahrensabbildung. Durch ihre Formalität und die Einfachheit der Syntax bzw. der zur Verfügung stehenden Symbole sind Petrinetze hochgradig flexibel und können auf fast jedes Anwendungsbeispiel angewandt werden. In der Stärke liegt jedoch auch die Schwäche, denn die Darstellung komplexerer Geschäftsprozesse wird sehr schnell sehr groß und damit auch unübersichtlich. Die beschränkte Anzahl an Symbolen und die einfache Möglichkeit der Abhängigkeiten (Graphen) erfordern eine aufwendige Abbildung von Prozessen, bei denen viele Inhalte miteinander kombiniert dargestellt werden müssen. In unserem Praxis-Beispiel erfordert die Abbildung der Terminabsprache des Bewerbungstermins z. B. im Vergleich zu BPMN deutlich mehr Platz und trägt damit zum flächenmäßigen Wachstum des Petrinetzes bei. Ein Beispiel dafür stellt die Information dar, dass die Absprache des Bewerbungstermins per E-Mail stattfindet. Solche Informationen können in Petrinetzen nur schriftlich hinterlegt werden. Die größte Herausforderung bei der Transformation eines EPKs in ein Petrinetz ist die Modellierung der Konnektoren (AND, OR, XOR). Diese können in Petrinetzen nicht eindeutig implementiert werden. Diesem Umstand ist es geschuldet, dass Petrinetze erweitert werden, aber dadurch jedoch ihre Formalität teilweise verlieren oder einfach nicht standardisiert sind.

\section{III.II BEWERTUNGSKRITERIEN}

Um die vorgestellten Methoden besser voneinander abgrenzen bzw. bewerten zu können, ist es notwendig, eindeutige Kriterien zu formulieren. In diesem Beitrag liegt der Fokus klar auf der Transformation und der anschließenden Automatisierbarkeit. Diese Fähigkeit ist dementsprechend eine der Hauptkriterien zur Unterscheidung der Methoden. Für die Weitergabe von Informationen ist es außerdem wichtig, ob eine Methode formal beschrieben ist und somit eine eindeutige Semantik und Syntax besitzt. Dies wurde ebenfalls als Kriterium festgehalten.

Da bei der Modellierung fachgebietsübergreifend gearbeitet wird und die Modelle an Ausprägung zunehmen, ist es wichtig, Übersichtlichkeit zu gewährleisten. Jenes fachgebiets- übergreifende Arbeiten ist auch ein Grund, warum ein Modell einfach in der Handhabung sein sollte. Jeder Anwender ist somit in der Lage, sowohl seinen als auch andere Prozesse zu verstehen und weiterzugeben. Eine Standardisierung durch eine offizielle Institution unterstützt die universelle Übertragbarkeit und bildet damit das letzte Unterscheidungskriterium in dieser Arbeit.

\section{III.III BEWERTUNGSMATRIX}

Die in den vorherigen Abschnitten ermittelten Vor- und Nachteile lassen sich in der folgenden Bewertungsmatrix (Abb. 3) übersichtlich darstellen. Dabei steht ein Plus für eine positive und ein Minus für eine negative $\mathrm{Be}$ wertung. Ist ein Kriterium in besonderer Ausprägung gegeben, so wird dieses durch doppelte Vergabe vom jeweiligen Symbol hervorgehoben.

Grundsätzlich lässt sich feststellen, dass die Transformation eines Geschäftsprozesses, der mit der EPKMethode modelliert wurde, in eine formale Methode möglich ist. Eine Transformation lässt sich bis zu einem gewissen Grade ohne Probleme durchführen. In vielen Fällen aber müssen auf der operativen Ebene mehr oder weniger Korrekturen vorgenommen werden.

So eignen sich S-BPM und bedingt auch Petrinetze für die Transformation zwischen fachlich-konzeptioneller und operativer Ebene. Dies ist vor allem darin begründet, dass beide Methoden ohne mögliche Konnektoren bzw. Gateways (vor allem OR und XOR) auskommen. Die Transformation ist damit wechselseitig, ebenenübergreifend durchführbar. Bei Petrinetzen bedarf es zusätzlich der Kontrolle einer Instanz, die dafür sorgt, dass es zu keinem Informationsverlust kommt. Jedoch führen bei beiden Methoden die fehlenden Konnektoren zu starker Unübersichtlichkeit. Eine verlustfreie Transformation einer EPK in ein vergleichbares BPMN-Modell ist generell zwar möglich, aber durch die verwendeten Gateways nicht immer rückwirkend durchführbar.

Die Methoden BPMN und EPK sind auf- 


\begin{tabular}{|c|c|c|c|c|c|}
\hline Methoden & EPK & S-BPM & UML & BPMN & Petrinetze \\
\hline Automatisierung möglich & - & + & - & + & + \\
\hline $\begin{array}{c}\text { Formalität gegeben } \\
\text { Übersichtlichkeit }\end{array}$ & - & + & - & + & + \\
\hline Einfache Handhabung & + & ++ & + & + & ++ \\
\hline Methode als Standard & - & - & + & + & + \\
\hline
\end{tabular}

Abb. 3) Bewertungsmatrix

grund ihrer einfachen syntaktischen Struktur auf fachlich-konzeptioneller Ebene vorzuziehen, da im Grunde jeder involvierte Akteur in der Lage ist, seine relevanten Prozesse abbilden zu können. Dabei stehen die beteiligten Akteure bei der Modellierung ihrer Prozesse in BPMN jedoch vor der Hürde einer mannigfaltigen Auswahl an Symbolen. Der augenscheinlich gravierendste Vorteil ist jedoch gleichzeitig ein Nachteil der EPK, denn bei komplexeren Darstellungen werden Verantwortungsbereiche nicht eindeutig dargestellt und müssen zusätzlich an anderer Stelle hinterlegt werden. Dies ist in BPMN anhand von Swimlanes und Pools möglich. Ein Versuch, diese Informationen auch in EPK zu hinterlegen, ist die Darstellung der Prozesse mit sogenannten Vorgangskettendiagrammen.

Ähnlich dazu gibt es auch in UML je nach Diagrammtyp (Aktivitätsdiagramm und Use-Case-Diagramm) eine Vielzahl unterschiedlicher Symbole, die das Modellieren erschweren. Diese Vielzahl kommt jedoch nur dann zum Tragen, wenn die im Prozess vorhandenen Parallelitäten von Akteuren so ausgeprägt sind, dass diese den eigentlichen Rahmen sprengen und zur Unübersichtlichkeit neigen. Diese Tatsache lässt sich bereits an dem relativ unkomplizierten Verfahren des Bewerbungsprozesses belegen. Für die Formalisierung gibt es keine festgelegten Standards, somit bietet die UML keine Möglichkeit der Automatisierung von Geschäftsprozessen. Es gibt Bemühungen von Forschungsgruppen, die UML in Petrinetze zu transformieren und somit eine Formalisierung zu ermöglichen, allerdings sind diese noch nicht Teil der eigentlichen Spezifikation.
Es ist festzustellen, dass Petrinetze aufgrund ihrer grundlegenden Struktur die Basis für alle heute eingesetzten Methoden bilden. Dementsprechend haben diese selbstverständlich weiterhin ihre Existenzberechtigung. Gleichzeitig sind jedoch die weiterentwickelten Methoden notwendig, um zusätzliche Informationen hinterlegen zu können, da bei steigendem Informationsgehalt die gleiche Übersichtlichkeit gewährleistet werden sollte.

Für Unternehmen ist es aus diesem Grunde gerade auf Führungsebene ratsam, sich mit allen Methoden zu beschäftigen, um die hier aufgeführkönnen. In erster Linie sollten sich Unternehmen bei der Wahl der Methode auf die fachlich-konzeptionelle Ebene stützen, da diese den Ausgangspunkt für sämtliches operatives Durchführen bildet. Eine Transformation bedarf stets der zusätzlichen Kommunikation, da es gerade hier zu möglichen Fehlern kommen kann.

\section{LITERATUR}

Alexandrow, L.; Geldmacher, J.; Geraniotaki, S.; Nikola P.; Wilhelm, A.; (2013): Transformation einer EPK in eine formalisierte Methode, Semesterarbeit zu Modellierung und Automatisierung von Geschäftsprozessen, Sommersemester 2013, Masterstudiengang Wirtschaftsinformatik, Fachbereich Betriebswirtschaft/Wirtschaftsinformatik, Technische Hochschule Wildau, Wildau

Bösing, K. D. (2013): BPMN als neuer Modellierungsstandard in: Wissenschaftliche Beiträge der TH Wildau, S. 121-127, Ungvári, L. (Hrsg.), Technische Hochschule Wildau, Wildau

Brocke, J. (2003): Referenzmodellierung - Gestaltung und Verteilung von Konstruktionsprozessen, Brocke, J (Hrsg.), Logos Verlag, Berlin;

Dandl, J. (1999): Objektorientierte Prozessmodellierung mit der UML und EPK, Arbeitspapiere WI, Nr.12, Johannes-Gutenberg-Universität Mainz, Mainz

Decker, G.; Tscheschner, W.; Puchan, J. (2009): Migration von EPK zu BPMN, in: EPK 2009, Geschäftsprozessmanagement mit Ereignisgesteuerten Prozessketten, Proceeding, S. 91-109, Nüttgens, M.; Rump; F. J.; Mendling, J.; Gehrke, N. (Hrsg.), 8. Workshop der Gesellschaft für Informatik e.V., Berlin ten Vor- und Nachteile abwägen zu
Eckleder, A. (2006): Semantikprüfung von Geschäftsprozessmodellen, http://193.196.7.212/woped/wpcontent/uploads/2013/01/STA_ECKLEDER.pdf, Zugriff: 21.05.2013

Gadatsch, A. (2010): Grundkurs Geschäftsprozess-Management, 6. Auflage, Vieweg+Teubner Verlag, Wiesbaden

Gehring, H.; Gadatsch, A. (1999): Ein Rahmenkonzept für die Modellierung von Geschäftsprozessen und Workflows, Fachbereichsbericht Nr. 274, Fachbereich Wirtschaftswissenschaft, FernUniversität Hagen, Hagen Kotsev, V.; Stanev, I; Grigorova, K. (2011): BPMN-EPCBPMN Converter, in: Imagination, Creativity, Design, Development, Proceeding of the International Students Conference on Informatics, ICDD, S. 150-156, Simian, D. (Ed.), Sibiu, Romania

Metasonic AG (2010): Case Study - Einführung von Geschäftsprozessmanagement mit Hilfe von subjektorientierten BPM (S BPM) bei der FIDUCIA, Pfaffenhofen, Metasonic AG (Hrsg.), http://www.metasonic.de/sites/ default/files/pdf/6/Metasonic_Fiducia_Case_Study_ de.pdf, Zugriff: 10.04.2013

Object Management Group (2011): Business Process Model and Notation (BPMN) Version 2.0, http://www. omg.org/spec/BPMN/2.0, Zugriff: 20.06.2013

\section{AUTOREN}

Prof. Dr. Klaus D. Bösing

Technische Hochschule Wildau

Fachbereich Wirtschaft, Informatik und Recht

Fachgebiet Software Engineering

Tel.: +493375 508952

eMail: klaus.boesing@th-wildau.de

Jan Geldmacher

Studiengang M. Sc. Wirtschaftsinformatik

Technische Hochschule Wildau

Fachbereich Wirtschaft, Informatik und Recht eMail: jan.geldmacher@th-wildau.de 